\title{
Copper-Catalyzed Synthesis of 1,2,4-Triazoles via Sequential Coupling and Aerobic Oxidative Dehydrogenation of Amidines
}

\author{
Hao Xu, ${ }^{\text {a,b }}$ Yuyang Jiang, ${ }^{\mathrm{c}} \mathrm{Hua} \mathrm{Fu}{ }^{* a, c}$ \\ a Key Laboratory of Bioorganic Phosphorus Chemistry and Chemical Biology (Ministry of Education), Department of Chemistry, \\ Tsinghua University, Beijing 100084, P. R. of China \\ Fax+86(10)62781695; E-mail: fuhua@mail.tsinghua.edu.cn \\ b School of Chemistry and Chemical Engineering, Henan University, Kaifeng 475001, P. R. of China \\ c Key Laboratory of Chemical Biology (Guangdong Province), Graduate School of Shenzhen, Tsinghua University, Shenzhen 518057, \\ P. R.of China
}

Received: 09.10.2012; Accepted after revision: 31.10.2012

\begin{abstract}
A convenient, efficient, and practical copper-catalyzed one-pot method for the synthesis of 1,2,4-triazoles has been developed via reactions of amidines. The procedure underwent sequential base-promoted intermolecular coupling (nucleophilic substitution) between two amidines and intramolecular aerobic oxidative dehydrogenation, and the inexpensive, convenient, and efficient method for the synthesis of 1,2,4-triazoles will attract much attention in academic and industrial research.
\end{abstract}

Key words: copper, aerobic oxidative, dehydrogenation, amidines, 1,2,4-triazoles

Nitrogen heterocycles occur widely in various natural products and biologically active molecules. ${ }^{1}$ The $1,2,4$-triazole derivatives are widely used in medicinal chemistry, materials science, and organocatalysis, and their synthesis has attracted much attention. ${ }^{2}$ The common methods are from intramolecular cyclizations of $N$-acylamidorazones that are prepared via couplings of hydrazines and carboxylic acid derivatives, ${ }^{3}$ but they often provide 1,2,4-triazoles in low yields. Therefore, it is highly desired to develop a simple and practical approach to 1,2,4-triazole derivatives. Recently, transition-metal-catalyzed aerobic oxidative formation of bonds is a focal field, ${ }^{4}$ and some nitrogen heterocycles, such as benzimidazoles, ${ }^{5}$ carbazoles, ${ }^{6}$ indazoles, ${ }^{7} \mathrm{~N}$-methoxylactams, ${ }^{8}$ and indolines, ${ }^{9}$ have been prepared via the aerobic oxidative strategy, in which expensive palladium-, rhodium-, and rutheniumbased catalysts are often necessary. During the past few years, there have been excellent progress in copper-catalyzed cross-couplings with inexpensive and low toxic copper-catalysts, and wide application with good functional tolerance has been explored. ${ }^{10,11}$ Recently, several efficient copper-catalyzed aerobic oxidative methods for the synthesis of nitrogen heterocycles have been developed by us ${ }^{12}$ and other groups. ${ }^{13}$ Nagasawa and coworkers have developed an efficient copper-catalyzed synthesis of 1,2,4-triazole derivatives via coupling of amidines with nitriles. ${ }^{14}$ Herein, we report a novel, convenient, and efficient copper-catalyzed one-pot synthesis of 1,2,4-tri-

SYNLETT 2013, 24, 0125-0129

Advanced online publication: 04.12.2012

DOI: 10.1055/s-0032-1317692; Art ID: ST-2012-W0864-L

(C) Georg Thieme Verlag Stuttgart · New York azoles via sequential coupling and aerobic oxidative dehydrogenation of amidines.

Reaction of benzamidine hydrochloride (1a) with cyclopropanecarboxamidine hydrochloride (1i) was used as the model to optimize reaction conditions including the catalysts, bases, solvents, temperature, and reaction time. As shown in Table 1, the copper-catalyzed one-pot synthesis of 3-cyclopropyl-5-phenyl-1 $H$-1,2,4-triazole (2i) underwent sequential two-step procedures: intermolecular coupling (nucleophilic substitution) between two amidines and intramolecular aerobic oxidative dehydrogenation. The first-step coupling was performed at $120{ }^{\circ} \mathrm{C}$ for 24 hours under $\mathrm{N}_{2}$ atmosphere, and the second step, the intramolecular formation of the $\mathrm{N}-\mathrm{N}$ bond, was carried out at $120{ }^{\circ} \mathrm{C}$ for 24 hours under $\mathrm{O}_{2}$. In order to prevent homogeneous coupling of benzamidine hydrochloride (1a; we found that aromatic amidines easily self-coupled), 1a was added $(3 \times 0.25 \mathrm{mmol})$ every eight hours. Seven copper catalysts ( 0.1 equiv) were screened by using two equivalents of $\mathrm{Cs}_{2} \mathrm{CO}_{3}$ as the base (relative to amount of 1i), and DMSO as the solvent (Table 1, entries 1-7), and $\mathrm{Cu}$ powder exhibited the highest activity (Table 1, entry 7). Only trace amount of target product was observed in the absence of copper catalyst (Table 1 , entry 8 ). Other bases were determined (Table 1, entries 9-12), and they were inferior to $\mathrm{Cs}_{2} \mathrm{CO}_{3}$ (compare entries 7, 9-12, Table 1). Affect of solvents was also investigated (compare entries 7, 13-15, Table 1), and DMSO provided the highest efficiency. We attempted different temperature (Table 1, entries 16 and 17), and $120^{\circ} \mathrm{C}$ was suitable (Table 1, compare entries 7, 16, and 17). The second step, the aerobic oxidative dehydrogenation, was elongated to 48 hours, and a higher yield was afforded (Table 1, entry 18). When the one-pot, two-step reaction was performed under $\mathrm{N}_{2}$ (Table 1, entry 19) or air (Table 1, entry 20), lower yields were provided. We changed amount of 1a (Table 1, entries 21 and 22), and the results showed that four equivalents of 1a (1a was added by ratio of 2:1:1) gave $\mathbf{2} \mathbf{i}$ in $72 \%$ yield (Table 1 , entry 22 ).

With the optimum reaction conditions in hand, the scope of the copper-catalyzed one-pot synthesis of 1,2,4-triazoles was investigated. As shown in Table 2, the examined substrates provided moderate to good yields. Aromatic amidines self-coupled to give homogeneous 
products (Table 2, entries 1-5). Heterogeneous reactions of aromatic amidines with aliphatic amidines were also performed well (Table 2, entries 6-19), but aromatic amidines were required to add to the system $(3 \times)$ by the ratio (2:1:1) every eight hours in order to prevent self-reaction of the aromatic amidines. In the copper-catalyzed reaction, no ligand or additive was needed. The reactions could tolerate some functional groups including $\mathrm{C}-\mathrm{Cl}$ bond (Table 2, entries 3,14-16), nitro (Table 2, entry 4), and N-heterocycle (Table 2, entries 5, 17-19) in the substrates.

Table 1 Copper-Catalyzed One-Pot Synthesis of 3-Cyclopropyl-5phenyl-1H-1,2,4-triazole (2i) via Reaction of Benzamidine Hydrochloride (1a) with Cyclopropanecarboxamidine Hydrochloride (1i): Optimization of Conditions ${ }^{\text {a }}$
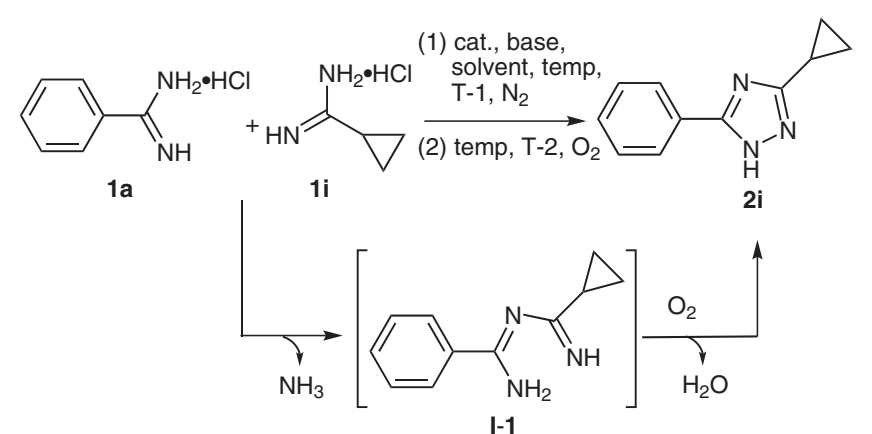

\begin{tabular}{|c|c|c|c|c|c|c|}
\hline Entry & Catalyst & Base & Solvent & $\begin{array}{l}\text { Temp } \\
\left({ }^{\circ} \mathrm{C}\right)\end{array}$ & $\begin{array}{l}\text { T-1 (h)/ } \\
\text { T-2 (h) }\end{array}$ & $\begin{array}{l}\text { Yield } \\
(\%)^{b}\end{array}$ \\
\hline 1 & $\mathrm{CuI}$ & $\mathrm{Cs}_{2} \mathrm{CO}_{3}$ & DMSO & 120 & $24 / 24$ & 31 \\
\hline 2 & $\mathrm{CuBr}$ & $\mathrm{Cs}_{2} \mathrm{CO}_{3}$ & DMSO & 120 & $24 / 24$ & 38 \\
\hline 3 & $\mathrm{CuCl}$ & $\mathrm{Cs}_{2} \mathrm{CO}_{3}$ & DMSO & 120 & $24 / 24$ & 38 \\
\hline 4 & $\mathrm{Cu}_{2} \mathrm{O}$ & $\mathrm{Cs}_{2} \mathrm{CO}_{3}$ & DMSO & 120 & $24 / 24$ & 33 \\
\hline 5 & $\mathrm{CuO}$ & $\mathrm{Cs}_{2} \mathrm{CO}_{3}$ & DMSO & 120 & $24 / 24$ & 27 \\
\hline 6 & $\mathrm{Cu}(\mathrm{OAc})_{2}$ & $\mathrm{Cs}_{2} \mathrm{CO}_{3}$ & DMSO & 120 & $24 / 24$ & 21 \\
\hline 7 & $\mathrm{Cu}$ & $\mathrm{Cs}_{2} \mathrm{CO}_{3}$ & DMSO & 120 & $24 / 24$ & 44 \\
\hline 8 & - & $\mathrm{Cs}_{2} \mathrm{CO}_{3}$ & DMSO & 120 & $24 / 24$ & trace \\
\hline 9 & $\mathrm{Cu}$ & $\mathrm{NaOAc}$ & DMSO & 120 & $24 / 24$ & 17 \\
\hline 10 & $\mathrm{Cu}$ & $\mathrm{K}_{2} \mathrm{CO}_{3}$ & DMSO & 120 & $24 / 24$ & 35 \\
\hline 11 & $\mathrm{Cu}$ & $\mathrm{K}_{3} \mathrm{PO}_{4}$ & DMSO & 120 & $24 / 24$ & 34 \\
\hline 12 & $\mathrm{Cu}$ & $\mathrm{KO} t-\mathrm{Bu}$ & DMSO & 120 & $24 / 24$ & 28 \\
\hline 13 & $\mathrm{Cu}$ & $\mathrm{Cs}_{2} \mathrm{CO}_{3}$ & dioxane & 120 & $24 / 24$ & 5 \\
\hline 14 & $\mathrm{Cu}$ & $\mathrm{Cs}_{2} \mathrm{CO}_{3}$ & $o$-xylene & 120 & $24 / 24$ & 34 \\
\hline 15 & $\mathrm{Cu}$ & $\mathrm{Cs}_{2} \mathrm{CO}_{3}$ & DMF & 120 & $24 / 24$ & 42 \\
\hline 16 & $\mathrm{Cu}$ & $\mathrm{Cs}_{2} \mathrm{CO}_{3}$ & DMSO & 90 & $24 / 24$ & trace \\
\hline 17 & $\mathrm{Cu}$ & $\mathrm{Cs}_{2} \mathrm{CO}_{3}$ & DMSO & 140 & $24 / 24$ & 38 \\
\hline 18 & $\mathrm{Cu}$ & $\mathrm{Cs}_{2} \mathrm{CO}_{3}$ & DMSO & 120 & $24 / 48$ & 52 \\
\hline 19 & $\mathrm{Cu}$ & $\mathrm{Cs}_{2} \mathrm{CO}_{3}$ & DMSO & 120 & 72 & $28^{c}$ \\
\hline 20 & $\mathrm{Cu}$ & $\mathrm{Cs}_{2} \mathrm{CO}_{3}$ & DMSO & 120 & 72 & $41^{\mathrm{d}}$ \\
\hline
\end{tabular}

Table 1 Copper-Catalyzed One-Pot Synthesis of 3-Cyclopropyl-5phenyl-1H-1,2,4-triazole (2i) via Reaction of Benzamidine Hydrochloride (1a) with Cyclopropanecarboxamidine Hydrochloride (1i): Optimization of Conditions ${ }^{\mathrm{a}}$ (continued)

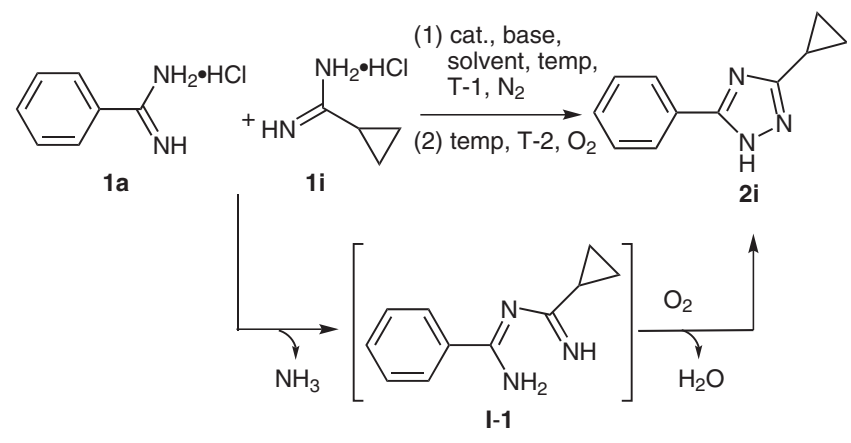

\begin{tabular}{lllllll}
\hline Entry & Catalyst & Base & Solvent & $\begin{array}{l}\text { Temp } \\
\left({ }^{\circ} \mathrm{C}\right)\end{array}$ & $\begin{array}{l}\mathrm{T}-1(\mathrm{~h}) / \\
\mathrm{T}-2(\mathrm{~h})\end{array}$ & $\begin{array}{l}\text { Yield } \\
(\%)^{\mathrm{b}}\end{array}$ \\
\hline 21 & $\mathrm{Cu}$ & $\mathrm{Cs}_{2} \mathrm{CO}_{3}$ & DMSO & 120 & $24 / 48$ & $64^{\mathrm{e}}$ \\
22 & $\mathrm{Cu}$ & $\mathrm{Cs}_{2} \mathrm{CO}_{3}$ & DMSO & 120 & $24 / 48$ & $72^{\mathrm{f}}$ \\
\hline
\end{tabular}

${ }^{a}$ Reaction conditions: benzamidine hydrochloride (1a, $3 \times 0.25$ mmol) was added $(3 \times)$ every $8 \mathrm{~h}$, cyclopropanecarboxamidine hydrochloride (1i, $0.5 \mathrm{mmol})$, catalyst $(0.1 \mathrm{mmol})$, base $(2 \mathrm{mmol})$, solvent $(1.5 \mathrm{~mL})$, under nitrogen atmosphere for the first step, under oxygen balloon (1 bar) for the second step.

${ }^{\mathrm{b}}$ Isolated yield.

${ }^{\mathrm{c}}$ Under $\mathrm{O}_{2}$ for the two steps.

${ }^{\mathrm{d}}$ Under air for the two steps.

${ }^{\mathrm{e}}$ Conditions: $1 \mathrm{a}(3 \times 0.5 \mathrm{mmol})$ and $\mathrm{Cs}_{2} \mathrm{CO}_{3}(2.5 \mathrm{mmol})$ were added $(3 \times)$ every $8 \mathrm{~h}$.

${ }^{\mathrm{f}}$ Conditions: $1 \mathrm{a}(1 \mathrm{mmol}+2 \times 0.5 \mathrm{mmol})$ and $\mathrm{Cs}_{2} \mathrm{CO}_{3}(3.0 \mathrm{mmol})$ were added $(3 \times)$ every $8 \mathrm{~h}$.

Table 2 Copper-Catalyzed One-Pot Synthesis of 1,2,4-Triazoles via Sequential Coupling and Aerobic Oxidative Dehydrogenation of Amidines $^{\mathrm{a}}$

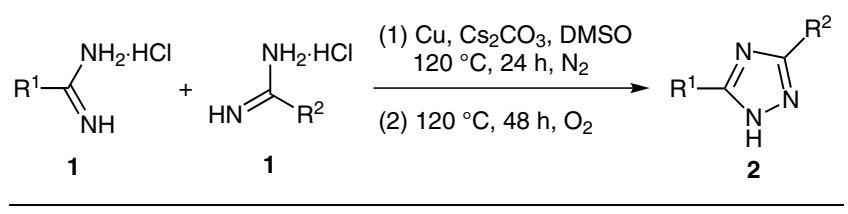

\begin{tabular}{lllllll}
\hline Entry & $\mathbf{1}$ & $\mathrm{R}^{1}$ & $\mathbf{1}$ & $\mathrm{R}^{2}$ & $\mathbf{2}$ & $\begin{array}{l}\text { Yield } \\
(\%)^{\mathrm{b}}\end{array}$ \\
\hline 1 & $\mathbf{1 a}$ & $\mathrm{Ph}$ & & $\mathbf{1 a}$ & $\mathrm{Ph}$ &
\end{tabular}

\begin{tabular}{rllllll}
\hline 1 & $\mathbf{1 a}$ & $\mathrm{Ph}$ & $\mathbf{1 a}$ & $\mathrm{Ph}$ & $\mathbf{2 a}$ & 62 \\
2 & $\mathbf{1 b}$ & $4-\mathrm{MeC}_{6} \mathrm{H}_{4}$ & $\mathbf{1 b}$ & $4-\mathrm{MeC}_{6} \mathrm{H}_{4}$ & $\mathbf{2 b}$ & 61 \\
3 & $\mathbf{1 c}$ & $4-\mathrm{ClC}_{6} \mathrm{H}_{4}$ & $\mathbf{1 c}$ & $4-\mathrm{ClC}_{6} \mathrm{H}_{4}$ & $\mathbf{2 c}$ & 60 \\
4 & $\mathbf{1 d}$ & $3-\mathrm{O}_{2} \mathrm{NC}_{6} \mathrm{H}_{4}$ & $\mathbf{1 d}$ & $3-\mathrm{O}_{2} \mathrm{NC}_{6} \mathrm{H}_{4}$ & $\mathbf{2 d}$ & 64 \\
5 & $\mathbf{1 e}$ & $4-\mathrm{pyridyl}$ & $\mathbf{1 e}$ & $4-\mathrm{pyridyl}$ & $\mathbf{2 e}$ & 53 \\
6 & $\mathbf{1 a}$ & $\mathrm{Ph}$ & $\mathbf{1 f}$ & $\mathrm{Me}$ & $\mathbf{2 f}$ & 80 \\
7 & $\mathbf{1 a}$ & $\mathrm{Ph}$ & $\mathbf{1 g}$ & $\mathrm{Et}$ & $\mathbf{2 g}$ & $45^{\mathrm{c}}$ \\
8 & $\mathbf{1 a}$ & $\mathrm{Ph}$ & $\mathbf{1 h}$ & $n-\mathrm{Pr}$ & $\mathbf{2 h}$ & $40^{\mathrm{c}}$ \\
9 & $\mathbf{1 a}$ & $\mathrm{Ph}$ & $\mathbf{1 i}$ & $c-\mathrm{Pr}$ & $\mathbf{2 i}$ & 72 \\
10 & $\mathbf{1 a}$ & $\mathrm{Ph}$ & $\mathbf{1 j}$ & $t-\mathrm{Bu}$ & $\mathbf{2 j}$ & 49
\end{tabular}


Table 2 Copper-Catalyzed One-Pot Synthesis of 1,2,4-Triazoles via Sequential Coupling and Aerobic Oxidative Dehydrogenation of Amidines $^{\mathrm{a}}$ (continued)

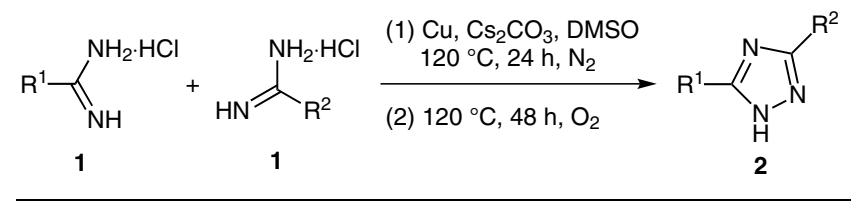

\begin{tabular}{lllllll}
\hline Entry & $\mathbf{1}$ & $\mathrm{R}^{1}$ & $\mathbf{1}$ & $\mathrm{R}^{2}$ & $\mathbf{2}$ & $\begin{array}{l}\text { Yield } \\
(\%)^{\mathrm{b}}\end{array}$ \\
\hline 11 & $\mathbf{1 b}$ & $4-\mathrm{MeC}_{6} \mathrm{H}_{4}$ & $\mathbf{1 f}$ & $\mathrm{Me}$ & $\mathbf{2 k}$ & 85 \\
12 & $\mathbf{1 b}$ & $4-\mathrm{MeC}_{6} \mathrm{H}_{4}$ & $\mathbf{1 g}$ & $\mathrm{Et}$ & $\mathbf{2 l}$ & $42^{\mathrm{c}}$ \\
13 & $\mathbf{1 b}$ & $4-\mathrm{MeC}_{6} \mathrm{H}_{4}$ & $\mathbf{1 i}$ & $c-\mathrm{Pr}$ & $\mathbf{2 m}$ & 91 \\
14 & $\mathbf{1 c}$ & $4-\mathrm{ClC}_{6} \mathrm{H}_{4}$ & $\mathbf{1 f}$ & $\mathrm{Me}$ & $\mathbf{2 n}$ & 75 \\
15 & $\mathbf{1 c}$ & $4-\mathrm{ClC}_{6} \mathrm{H}_{4}$ & $\mathbf{1 i}$ & $c-\mathrm{Pr}$ & $\mathbf{2 o}$ & 78 \\
16 & $\mathbf{1 c}$ & $4-\mathrm{ClC}_{6} \mathrm{H}_{4}$ & $\mathbf{1 j}$ & $t$-Bu & $\mathbf{2 p}$ & 51 \\
17 & $\mathbf{1 e}$ & $4-p y r i d y l$ & $\mathbf{1 f}$ & $\mathrm{Me}$ & $\mathbf{2 q}$ & 70 \\
18 & $\mathbf{1 e}$ & $4-p y r i d y l$ & $\mathbf{1 i}$ & $c-\mathrm{Pr}$ & $\mathbf{2 r}$ & 84 \\
19 & $\mathbf{1 e}$ & $4-p y r i d y l$ & $\mathbf{1 j}$ & $t$-Bu & $\mathbf{2 s}$ & 86 \\
\hline
\end{tabular}

${ }^{\text {a }}$ Reaction conditions: amidine-1 + amidine-2 $(1.0 \mathrm{mmol})$ for entries 1-5, amidine-1 $(2.0 \mathrm{mmol})$ for entries $6-19$ [added $(3 \times: 1 \mathrm{mmol}+2 \times$ $0.5 \mathrm{mmol})$ every $8 \mathrm{~h}$ ], amidine-2 $(0.5 \mathrm{mmol})$ for entries $6-19, \mathrm{Cu}$ powder $(0.1 \mathrm{mmol}), \mathrm{Cs}_{2} \mathrm{CO}_{3}(1.5 \mathrm{mmol}$ for entries $1-5 ; 3.0 \mathrm{~mol}$ for entries 6-19), DMSO $(1.5 \mathrm{~mL})$, reaction temperature $\left(120^{\circ} \mathrm{C}\right)$, reaction time ( $24 \mathrm{~h}$ for the first step; $48 \mathrm{~h}$ for the second step), under nitrogen atmosphere for the first step, under oxygen balloon ( 1 bar) for the second step.

${ }^{\mathrm{b}}$ Isolated yield.

${ }^{\mathrm{c}}$ Conditions: $0.5 \mathrm{~mL} t$-BuOH were added.

We explored the reaction mechanism for the synthesis of 1,2,4-triazoles. As shown in Scheme 1, treatment of 4methylbenzamidine hydrochloride (1b) was first carried out in the presence of $\mathrm{Cs}_{2} \mathrm{CO}_{3}$ in DMSO under $\mathrm{N}_{2}$ (no addition of $\mathrm{Cu}$ powder), and $N$-[amino( $m$-tolyl)methylene]4-methylbenzamidine (I-2) was obtained in 44\% yield (I2 was purified by recrystallization which led to the loss of some product because of its high polarity, Scheme 1, i).
The synthesized $N$-[amino( $m$-tolyl)methylene]-4-methylbenzamidine was treated in the presence of $\mathrm{Cu}$ powder under $\mathrm{O}_{2}$, and the target product $\mathbf{2 b}$ was provided in $68 \%$ yield (Scheme 1, ii). Therefore, a possible mechanism for the synthesis of 1,2,4-triazoles is proposed in Scheme 2. Amidine hydrochlorides transformed into free amidines in the presence of base $\left(\mathrm{Cs}_{2} \mathrm{CO}_{3}\right)$, and intermolecular nucleophilic attack of amino in one amidine to carbon in another one leads to intermediate I. Treatment of $\mathbf{I}$ with copper in the presence of $\mathrm{O}_{2}$ provides $\mathrm{Cu}$ (III) complex II (the similar metal complexes have been reported in the previous literature ${ }^{15}$ ), and reductive elimination of II affords the target product $(\mathbf{2})^{14}$ leaving $\mathrm{Cu}(\mathrm{I})$ complex III. Further, reaction of III with I regenerates II, and the target product $\mathbf{2}^{16}$ continuously is provided in the catalytic cycle.

In summary, we have developed a convenient, efficient, and practical copper-catalyzed one-pot method for the synthesis of 1,2,4-triazoles. The protocol uses readily available substituted amidines as the starting materials, inexpensive $\mathrm{Cu}$ powder as the catalyst, and economical and environment friendly oxygen as the oxidant, and the corresponding 1,2,4-triazoles were obtained in moderate to good yields. The procedure underwent sequential basepromoted intermolecular coupling (nucleophilic substitution) between two amidines and intramolecular aerobic oxidative dehydrogenation, and the inexpensive, convenient, and efficient method for the synthesis of 1,2,4-triazoles will attract much attention in academic and industrial researches because of the wide application of these compounds in various fields.

\section{Acknowledgment}

The authors wish to thank the National Natural Science Foundation of China (Grant Nos. 20972083 and 21172128), and the Ministry of Science and Technology of China (Grant No. 2012CB722605) for financial support.

Supporting Information for this article is available online at http://www.thieme-connect.com/ejournals/toc/synlett.

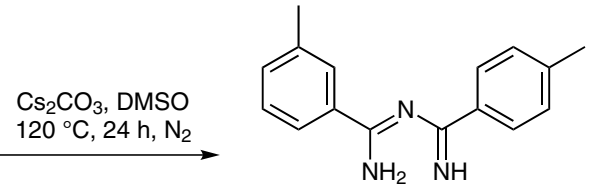

I-2 (44\% yield)

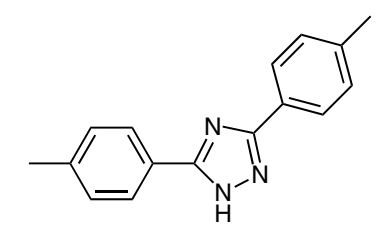

$2 \mathbf{b}(68 \%$ yield)

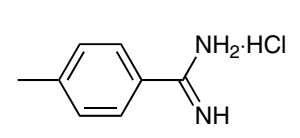

$1 b$

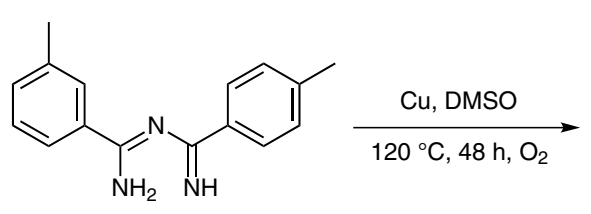

I-2
Scheme 1 (i) Treatment of 4-methylbenzamidine hydrochloride (1b) in the presence of $\mathrm{Cs}_{2} \mathrm{CO}_{3}$ in DMSO under $\mathrm{N}_{2}$ leading to $\mathrm{N}$-[amino(3tolyl)methylene]-4-methylbenzamidine (I-2); (ii) copper-catalyzed aerobic oxidation of $\mathbf{I}-2$ leading to $\mathbf{2 b}$ in the presence of $\mathrm{Cu}_{\mathrm{u}}$ powder under $\mathrm{O}_{2}$ 


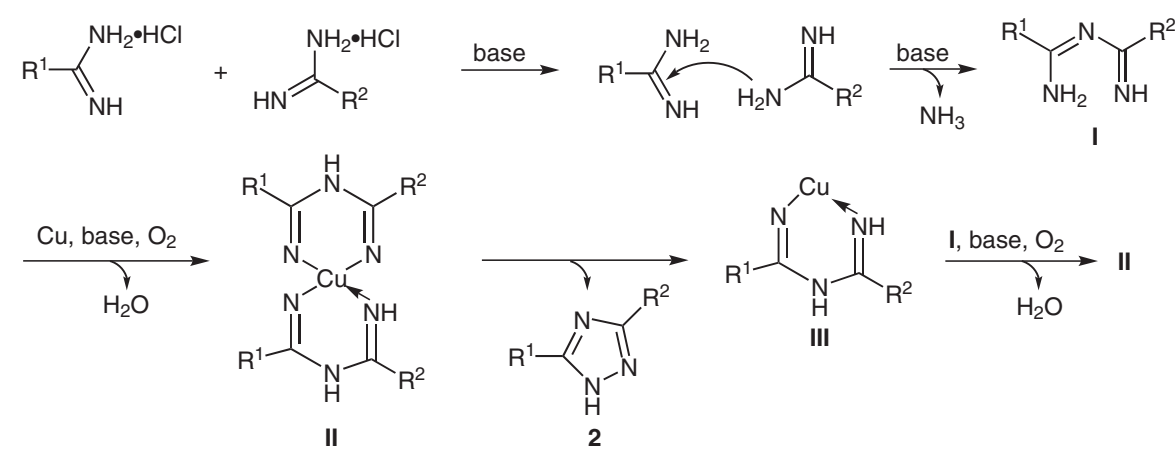

Scheme 2 Possible mechanism for synthesis of 1,2,4-triazoles

\section{References and Notes}

(1) (a) DeSimone, R. W.; Currie, K. S.; Mitchell, S. A.; Darrow, J. W.; Pippin, D. A. Comb. Chem. High Throughput Screening 2004, 7, 473. (b) Leeson, P. D.; Springthorpe, B. Nat. Rev. Drug Discovery 2007, 6, 881.

(2) (a) Al-Masoudi, I. A.; Al-Soud, Y. A.; Al-Salihi, N. J.; AlMasoudi, N. A. Chem. Heterocycl. Compd. (N.Y.) 2006, 42, 1377. (b) Huntsman, E.; Balsells, J. Eur. J. Org. Chem. 2005, 3761 .

(3) (a) Larsen, S. D.; DiPaolo, B. A. Org. Lett. 2001, 3, 3341. (b) Stocks, M. J.; Cheshire, D. R.; Reynold, R. Org. Lett. 2004, 6, 2969. (c) Balsells, J.; DiMichele, L.; Liu, J.; Kubryk, M.; Hansen, K.; Armstrong, J. D. III. Org. Lett. 2005, 7, 1039.

(4) For some reviews, see: (a) Stahl, S. S. Angew. Chem. Int. Ed. 2004, 43, 3400. (b) Punniyamurthy, T.; Velusamy, S.; Iqbal, J. Chem. Rev. 2005, 105, 2329. (c) Shi, Z.; Zhang, C.; Tang, C.; Jiao, N. Chem. Soc. Rev. 2012, 41, 3381. (d) Campbell, A. N.; Stahl, S. S. Acc. Chem. Res. 2012, 45, 851.

(e) Wendlandt, A. E.; Suess, A. M.; Stahl, S. S. Angew. Chem. Int. Ed. 2011, 50, 11062.

(5) Xiao, Q.; Wang, W.-H.; Liu, G.; Meng, F.-K.; Chen, J.-H.; Yang, Z.; Shi, Z.-J. Chem.-Eur. J. 2009, 15, 7292.

(6) (a) Tsang, W. C. P.; Zheng, N.; Buchwald, S. L. J. Am. Chem. Soc. 2005, 127, 14560. (b) Tsang, W. C. P.; Munday, R. H.; Brasche, G.; Zheng, N.; Buchwald, S. L. J. Org. Chem. 2008, 73, 7603. (c) Jordan-Hore, J. A.; Johansson, C. C. C.; Gulias, M.; Beck, E. M.; Gaunt, M. J. J. Am. Chem. Soc. 2008, 130, 16184.

(7) Inamoto, K.; Saito, T.; Katsuno, M.; Sakamoto, T.; Hiroya, K. Org. Lett. 2007, 9, 2931.

(8) Wasa, M.; Yu, J.-Q. J. Am. Chem. Soc. 2008, 130, 14058.

(9) (a) Mei, T.-S.; Wang, X.; Yu, J.-Q. J. Am. Chem. Soc. 2009, 131, 10806. (b) Neumann, J. J.; Rakshit, S.; Dröge, T.; Glorius, F. Angew. Chem. Int. Ed. 2009, 48, 6892.

(10) For recent reviews on copper-catalyzed cross-couplings, see: (a) Kunz, K.; Scholz, U.; Ganzer, D. Synlett 2003, 2428. (b) Ley, S. V.; Thomas, A. W. Angew. Chem. Int. Ed. 2003, 42, 5400. (c) Beletskaya, I. P.; Cheprakov, A. V. Coord. Chem. Rev. 2004, 248, 2337. (d) Evano, G.; Blanchard, N.; Toumi, M. Chem. Rev. 2008, 108, 3054. (e) Ma, D.; Cai, Q. Acc. Chem. Res. 2008, 41, 1450. (f) Monnier, F.; Taillefer, M. Angew. Chem. Int. Ed. 2009, 48, 6954. (g) Surry, D. S.; Buchwald, S. L. Chem. Sci. 2010, 1, 13. (h) Rao, H.; Fu, H. Synlett 2011, 745. (i) Liu, T.; Fu, H. Synthesis 2012, 44, 2805; and references cited therein.

(11) For selected papers, see: (a) Klapars, A.; Antilla, J. C.; Huang, X.; Buchwald, S. L. J. Am. Chem. Soc. 2001, 123, 7727. (b) Klapars, A.; Huang, X. H.; Buchwald, S. L. J. Am. Chem. Soc. 2002, 124, 7421. (c) Antilla, J. C.; Klapars, A.;
Buchwald, S. L. J. Am. Chem. Soc. 2002, 124, 11684. (d) Okano, K.; Tokuyama, H.; Fukuyama, T. Org. Lett. 2003, 5, 4987. (e) Gujadhur, R. K.; Bates, C. G.; Venkataraman, D. Org. Lett. 2001, 3, 4315. (f) Gajare, A. S.; Toyota, K.; Yoshifuji, M.; Yoshifuji, F. Chem. Commun. 2004, 1994. (g) Ma, D.; Zhang, Y.; Yao, J.; Wu, S.; Tao, F. J. Am. Chem. Soc. 1998, 120, 12459. (h) Ma, D.; Cai, Q.; Zhang, H. Org. Lett. 2003, 5, 2453. (i) Zhu, L.; Cheng, L.; Zhang, Y.; Xie, R.; You, J. J. Org. Chem. 2007, 72, 2737. (j) Rao, H.; Jin, Y.; Fu, H.; Jiang, Y.; Zhao, Y. Chem.-Eur. J. 2006, 12, 3636. (k) Guo, X.; Rao, H.; Jin, Y.; Fu, H.; Jiang, Y.; Zhao, Y. Adv. Synth. Catal. 2006, 348, 2197. (1) Jiang, D.; Fu, H.; Jiang, Y.; Zhao, Y. J. Org. Chem. 2007, 72,672 .

(12) (a) Wang, C.; Li, S.; Liu, H.; Jiang, Y.; Fu, H. J. Org. Chem. 2010, 75, 7936. (b) Lu, J.; Jin, Y.; Liu, H.; Jiang, Y.; Fu, H. Org. Lett. 2011, 13, 3694. (c) Xu, W.; Jin, Y.; Liu, H.; Jiang, Y.; Fu, H. Org. Lett. 2011, 13, 1274. (d) Xu, W.; Fu, H. J. Org. Chem. 2011, 76, 3846. (e) Xu, H.; Fu, H. Chem.-Eur. J. 2012, 18, 1180. (f) Wang, X.; Jin, Y.; Zhao, Y.; Zhu, L.; $\mathrm{Fu}$, H. Org. Lett. 2012, 14, 452.

(13) (a) Brasche, G.; Buchwald, S. L. Angew. Chem. Int. Ed. 2008, 47, 1932. (b) Ueda, S.; Nagasawa, H. Angew. Chem. Int. Ed. 2008, 47, 6411. (c) Saha, P.; Ramana, T.; Purkait, N.; Ali, M. A.; Paul, R.; Punniyamurthy, T. J. Org. Chem. 2009, 74, 8719. (d) Wang, H.; Wang, Y.; Liang, D.; Liu, L.; Zhang, J.; Zhu, Q. Angew. Chem. Int. Ed. 2011, 50, 5677. (e) Wang, Y.-F.; Chen, H.; Zhu, X.; Chiba, S. J. Am. Chem. Soc. 2012, 134, 11980.

(14) Ueda, S.; Nagasawa, H. J. Am. Chem. Soc. 2009, 131, 15080.

(15) (a) Häger, I.; Fröhlich, R.; Würthwein, E.-U. Eur. J. Inorg. Chem. 2009, 2415. (b) Wikstrom, J. P.; Filatov, A. S.; Rybak-Akimova, E. V. Chem. Commun. 2010, 46, 424. (c) Kopylovich, M. N.; Pombeiro, A. J. L.; Fischer, A.; Kloo, L.; Kukushkin, V. Y. Inorg. Chem. 2003, 42, 7239.

(16) General Procedure for the Synthesis of Compounds $2 a-s$ A $10 \mathrm{~mL}$ Schlenk tube was charged with a magnetic stirrer and DMSO $(1.5 \mathrm{~mL})$. For entries $1-5$ in Table 2 , aromatic amidine ( $1 \mathrm{mmol})$, Cu powder $(0.1 \mathrm{mmol}, 6.4 \mathrm{mg})$, and $\mathrm{Cs}_{2} \mathrm{CO}_{3}(2 \mathrm{mmol}, 489 \mathrm{mg}$ ) were added to the tube. The mixture was stirred at $120^{\circ} \mathrm{C}$ for $24 \mathrm{~h}$ under nitrogen atmosphere, and then the nitrogen atmosphere was changed into oxygen atmosphere (other conditions were kept). The following aerobic oxidative intramolecular formation of $\mathrm{N}-$ $\mathrm{N}$ bond was carried out at $120^{\circ} \mathrm{C}$ for $48 \mathrm{~h}$. The resulting mixture was cooled to r.t. and filtered, and the solid was washed with EtOAc $(3 \times 3 \mathrm{~mL})$. The combined filtrate was concentrated by a rotary evaporator, and the residue was purified by column chromatography on silica gel using PEEtOAc as eluent to give the desired target product. For entries 6-19 in Table 2, aromatic amidine $(1.0 \mathrm{mmol})$, 
aliphatic amidine $(0.5 \mathrm{mmol}), \mathrm{Cu}$ powder $(0.1 \mathrm{mmol}, 6.4$ $\mathrm{mg})$, and $\mathrm{Cs}_{2} \mathrm{CO}_{3}(3.0 \mathrm{mmol}, 978 \mathrm{mg})$ were added to the tube. The mixture was stirred at $120^{\circ} \mathrm{C}$ under nitrogen atmosphere, and additional aromatic amidine $(2 \times 0.5 \mathrm{mmol})$ was added to the resulting solution after $8 \mathrm{~h}$ and $16 \mathrm{~h}$, respectively. The reaction was performed for a total $24 \mathrm{~h}$ under nitrogen atmosphere, and then the nitrogen atmosphere was changed into oxygen atmosphere (other conditions were kept). The following aerobic oxidative intramolecular formation of $\mathrm{N}-\mathrm{N}$ bond was carried out at $120{ }^{\circ} \mathrm{C}$ for $48 \mathrm{~h}$. The workup procedure was similar to that of entries $1-5$ in Table 2. Data for three representative examples are given here.

3-Methyl-5-phenyl-4H-1,2,4-triazole (2f $)^{14}$

Eluent: PE-EtOAc (1:1); yield $64 \mathrm{mg}(80 \%)$; white solid; mp 163-165 ${ }^{\circ} \mathrm{C}\left(\right.$ lit. $\left..{ }^{14} \mathrm{mp} 163-165^{\circ} \mathrm{C}\right) .{ }^{1} \mathrm{H}$ NMR $(600 \mathrm{MHz}$, DMSO- $\left.d_{6}\right): \delta=13.75(\mathrm{~s}, 1 \mathrm{H}), 7.95(\mathrm{~d}, 2 \mathrm{H}, J=7.56 \mathrm{~Hz})$, 7.44-7.33 (m, $3 \mathrm{H}), 2.35$ (s, $3 \mathrm{H}) .{ }^{13} \mathrm{C}$ NMR $(150 \mathrm{MHz}$, DMSO- $\left.d_{6}\right): \delta=160.8,154.3,131.7,129.3,129.1,126.2$, 126.1, 12.5. ESI-MS: $m / z=160.3[\mathrm{M}+\mathrm{H}]^{+} ; m / z=182.2[\mathrm{M}+\mathrm{Na}]^{+}$.
3-(4-Chlorophenyl)-5-cyclopropyl-4H-1,2,4-triazole (20) $)^{14}$

Eluent: PE-EtOAc (6:1); yield $85 \mathrm{mg}(78 \%)$; white solid; mp 203-205 ${ }^{\circ} \mathrm{C}$ (lit..$\left.^{14} \mathrm{mp} \mathrm{202-203}{ }^{\circ} \mathrm{C}\right) .{ }^{1} \mathrm{H}$ NMR $(600 \mathrm{MHz}$, DMSO- $\left.d_{6}\right): \delta=13.71(\mathrm{~s}, 1 \mathrm{H}), 7.91(\mathrm{~d}, 2 \mathrm{H}, J=8.9 \mathrm{~Hz})$, $7.57-7.40$ (m, $2 \mathrm{H}), 2.09-1.96(\mathrm{~m}, 1 \mathrm{H}), 1.06-0.80(\mathrm{~m}, 4 \mathrm{H})$. ${ }^{13} \mathrm{C}$ NMR $\left(150 \mathrm{MHz}, \mathrm{DMSO}-d_{6}\right): \delta=160.2,160.1,133.7$, 131.0, 129.2, 127.9, 8.6, 7.5. ESI-MS: $m / z=220.2[\mathrm{M}+\mathrm{H}]^{+}$; $m / z=242.0[\mathrm{M}+\mathrm{Na}]^{+}$.

4-(5-Methyl-4H-1,2,4-triazol-3-yl)pyridine (2q) ${ }^{17}$

Eluent: PE-EtOAc (4:1); yield $56 \mathrm{mg}(70 \%)$; white solid; mp 104-106 ${ }^{\circ} \mathrm{C}$ (lit. $\left.{ }^{17} \mathrm{mp} 207-209^{\circ} \mathrm{C}\right) .{ }^{1} \mathrm{H}$ NMR $(600 \mathrm{MHz}$, DMSO- $\left.d_{6}\right): \delta=13.94(\mathrm{~s}, 1 \mathrm{H}), 8.81-8.55(\mathrm{~m}, 2 \mathrm{H}), 7.91(\mathrm{~d}$, $2 \mathrm{H}, J=3.4 \mathrm{~Hz}$ ), 2.44 (s, $3 \mathrm{H}) .{ }^{13} \mathrm{C}$ NMR (150 MHz, DMSO$\left.d_{6}\right): \delta=159.4,154.8,150.8,139.1,120.5,12.2$. ESI-MS: $m / z$ $=161.2[\mathrm{M}+\mathrm{H}]^{+} ; m / z=183.1[\mathrm{M}+\mathrm{Na}]^{+}$.

(17) Lipinski, C. A.; Lamattina, J. L.; Oates, P. J. J. Med. Chem. 1986, 29, 2154. 